\title{
Holographisches Messverfahren zur dreidimensionalen Analyse von Aerosolen
}

\author{
Felix Frischkorn, Horst Kreitlow, Lothar Martini, Jörn Miesner, Bernd Stark, Roland Wernecke
}

\section{Einleitung und Problemstellung}

Aerosolpartikel sind Teilchen, die überall in der Erdatmosphäre präsent sind; sie können fest, fest/flüssig oder flüssig sein sowie von unterschiedlicher Größe $(0,001$ bis $100 \mu \mathrm{m}$ Durchmesser) und verschiedener chemischer Zusammensetzung [1].

Aerosolpartikel werden auf natuirliche Weise, z. B. bei Vulkanausbrüichen, großflächigen Bränden oder Stürmen, direkt oder indirekt und durch Einwirkungen des Menschen z. B. bei der industriellen Produktion, Landnutzungsänderungen und landwirtschaftlichen Tätigkeiten, in die Atmosphäre emittiert oder sie werden durch Gas-Partikelumwandlungen in-situ gebildet [2, 3, 4].

Ohne Aerosolkondensationskerne (CCN - Cloud condensation nuclei) wäre die Bildung von Wolkentröpfchen (Hydrometeore) in der Troposphäre nicht möglich. Aerosolpartikel und Wolkentröpfchen streuen und absorbieren Sonnenstrahlung und haben somit großen Einfluss auf die Strahlungsbilanz der Erdatmosphäre und damit auf das regionale und globale Klima der Erde.

Eine besonders große Wirkung von troposphärischen Aerosolteilchen auf das Klima ist durch ihre Rolle bei der Wolkenbildung und bei der Bildung von Niederschlag gegeben. Mit der Erhöhung der Aerosolteilchendichte ist nicht nur eine verstärkte Wolkenbildung verbunden, sondern auch eine Verschiebung des Tröpfchengrößenspektrums zu kleineren Tröpfchendurchmessern. Damit vergrößert sich die Strahlungsreflexion an der Wolkenobergrenze und fuihrt so zu einer partiellen Abkühlung der Erdoberfläche. Dieser Effekt kann allerdings nach derzeitigen Erkenntnissen den anthropogenen Treibhauseffekt, der als Folge des Anstiegs der Konzentration der „Treibhausgase“ in der Troposphäre auftritt, nur in einigen Gebieten der nördlichen Hemisphäre der Erde teilweise kompensieren [5, 6].

Interessant ist in diesem Zusammenhang die Frage, in welcher Weise sich die Bewölkung insgesamt als Folge der durch eine mögliche Klimaänderung veränderten dynamischen Bedingungen neu einstellen wird.

Um die Einflüsse von Aerosolen auf den Klimaantrieb in den Klimamodellen ausreichend zu berücksichtigen und damit verlässliche Voraussagen über die Entwicklung des regionalen und globalen Klimas treffen zu können, sind genaue Kenntnisse der dreidimensionalen Verteilungen und der optischen Eigenschaften der Aerosolpartikel und der Wolkentröpfchen Grundvoraussetzung. Da das grundsätzliche Verständnis auf diesem Gebiet heute noch als sehr lïckenhaft charakterisiert werden muss und nicht im erforderlichen Umfang vorliegt, ist die Problematik der Wechselwirkungen zwischen Aerosolen, Wolken und Klima
Gegenstand nationaler und internationaler Forschungsanstrengungen.

Das Bundesministerium für Bildung, Wissenschaft, Forschung und Technologie (BMBF) hat diesem Tatbestand Rechnung getragen und das Aerosol-Verbundforschungsvorhaben initiiert.

Die Deutsche Bundesstiftung Umwelt (DBU) fördert seit 1998 in einem Großverbund „Atmosphärische Diagnostik" die Entwicklung neuer Analysenmesstechniken, die kommerziell nicht verfügbar sind. Unter anderem wird in diesem Rahmen auch ein Projekt zur Entwicklung eines Messsystems zur „Dreidimensionalen Aufnahme von Aerosolverteilungen“" gefördert. Die Leitung dieses Projektes wurde der Technischen Fachhochschule Wildau übertragen. Kooperationspartner sind das Institut für Lasertechnik der Fachhochschule Ostfriesland Emden und die Firma deka Sensor \& Technologie Teltow.

Ziel des Projektes ist die Entwicklung eines mobilen Messgerätes, das nach einem holographischen Verfahren dreidimensionale Momentaufnahmen der Partikelverteilung in schneller Folge und damit auch Untersuchungen der Partikeldynamik gestattet. Das Projekt findet im Oktober 2000 bei einer Felderprobung des Messsystems auf dem Kleinen Feldberg im Taunus unter rauen Umweltbedingungen im Rahmen des Verbundes „Atmosphärische Diagnostik" seinen Abschluss.

Über die Grundlagen der Entwicklung des Projektes „Dreidimensionale Aufnahme von Aerosolverteilungen“ und das Ergebnis der Arbeiten soll im Folgenden berichtet werden.

Um Größenverteilungen von Aerosolpartikeln und Wolkentröpfchen messen zu können, wurden bisher Messgeräte eingesetzt, die auf der Basis der Streuung von Laserstrahlung arbeiten. Ein optischer Partikelzähler FSSP-100 (Forward Scattering Spektrometer Probe) ist ein Beispiel fuir ein solches Gerät [7]. Mit FSSP-Geräten ist es jedoch nicht möglich, dreidimensionale Aufnahmen von Aerosolverteilungen zu erhalten.

Dreidimensionale oder so genannte Volumenobjekte sind Gegenstand der Untersuchungen in verschiedenen Technik- und Wissenschaftsbereichen, wie z. B. der Partikeldiagnostik und der Mikrobiologie. Dabei ist das Verhalten von einzelnen Volumenbereichen im Gesamtvolumen von Interesse, wie z. B. das Wachsen von Kristallen bei der Kristallzüchtung oder das Größerwerden von Tumoren in der Medizin sowie die Untersuchung von Schadstoffpartikeln in der Luft. Für die Untersuchung ist die Aufzeichnung des Gesamtvolumens und die spätere schichtweise Auswertung der Teilvolumina erforderlich. Bei den genannten Forschungsaufgaben wird seit vielen Jahren die Holographie erfolgreich eingesetzt 
$[8,9]$. Bisher wurden dabei so genannte filmbasierte holographische Aufzeichnungsverfahren angewandt, wie es der klassischen Holographie entspricht. Die Hologrammanalyse ist bei diesen Aufzeichnungspraktiken bei Untersuchungsobjekten im Mikrometergrößenbereich mit einem enormen Zeitaufwand verbunden. Aus diesem Grund sind quasi Online-Aufzeichnungen nicht möglich. Diese sind jedoch erforderlich, um dynamische Wechselwirkungen zwischen Aerosolen, Wolken und Klima zu erforschen.

Die bisher eingesetzten Messeinrichtungen wurden auch weder miniaturisiert noch als mobile Messstationen für den Außeneinsatz realisiert. Im Gegensatz zu den bisher verwendeten filmbasierten Aufzeichnungsverfahren soll im Projekt „Dreidimensionale Aufnahme von Aerosolverteilungen“ ein neuartiges miniaturisiertes System mit einer CCD-Kamera zur in-situ Volumenbeobachtung und Aufzeichnung in Verbindung mit einem Bildverarbeitungssystem entwickelt werden.

\section{Grundlagen}

\subsection{Prinzip der Holographie}

Die Fotografie liefert nur Information über die Amplitude der Wellen. Information über die Phasenunterschiede der Wellen, in denen die Information über die Räumlichkeit des Objektes steckt, ist nicht vorhanden. Die Holographie enthält die gesamte Information über das Objekt, wie es sich einem Betrachter darstellt, der das Objekt aus einem Blickwinkel sieht, unter dem die Hologrammplatte oder die Kamera zum Objekt steht.

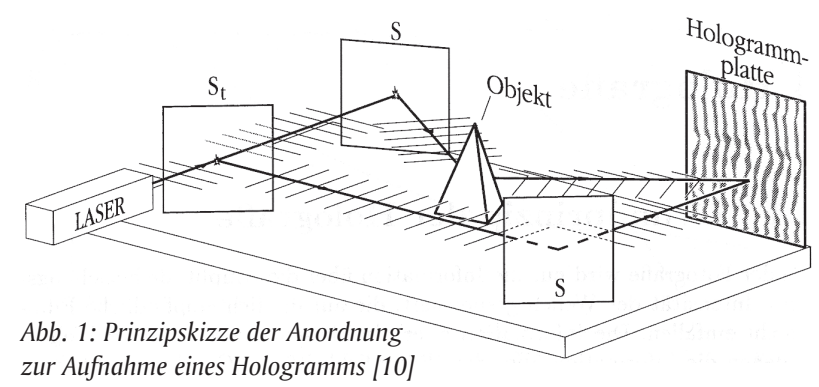

In der Abb. 1 ist schematisch eine Anordnung zur Aufnahme eines Hologramms dargestellt [10]. Als Objekt ist eine Pyramide gewählt. Diese Pyramide wird mit Hilfe eines Lasers mit kohärentem Licht bestrahlt. Das vom Objekt in die Richtung der Hologrammplatte reflektierte Licht ist die so genannte „Objektwelle“ der Feldstärke $E_{0}$. Die Phasenunterschiede der Teilwellen erhält man durch eine Referenzphase, die durch eine am Objekt vorbeilaufende „Referenzwelle“ der Feldstärke $E_{R}$ erzeugt wird. Am Ort

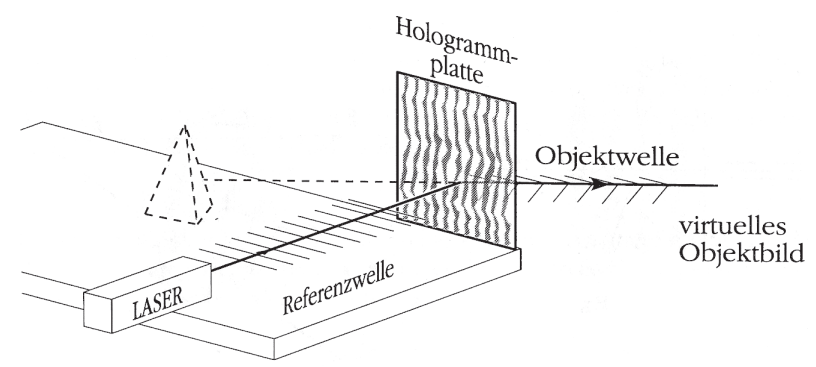

Abb. 2: Prinzipskizze der Anordnung zur Rekonstruktion eines Hologramms [10]

der Hologrammplatte werden Objekt- und Referenzwelle überlagert und können bei gleicher Polarisationsrichtung interferieren. Die entstehende Interferenzstruktur ist das so genannte Hologramm, das die Information zur Amplitude und Phase zum Objekt enthält. Dabei ist es wichtig, dass die beiden Teilstrahlen kohärent sind und aus derselben Laser-Strahlungsquelle stammen, was man durch die Verwendung einer Strahlteilerplatte hinter dem Laser erreicht. Im Interesse eines maximalen Kontrastes der Interferenzstruktur sollten die beiden Teilstrahlen im Bereich der Überlagerung ähnliche Intensität besitzen. Das erreicht man durch eine geeignete Dimensionierung der Strahlteilerplatte, die so gewählt sein sollte, dass die Intensität der uiber das Objekt laufenden Teilstrahlung beträchtlich höher ist als die der Referenzstrahlung. Zur Rekonstruktion der Information über das Objekt wird das entstandene Hologramm mit der Referenzstrahlung beleuchtet (Abb. 2) [10]. Dabei entsteht eine Welle, die vom ursprünglichen Objekt herzukommen scheint. Das Resultat ist das virtuelle bzw. reelle dreidimensionale Volumenbild des holographierten Objektes. Besteht das Objekt aus einem Volumen, in dem sich z. B. ein zu untersuchendes Aerosol mit einer solchen Dichte befindet, dass neben der an Aerosolteilchen gebeugten Strahlung auch Strahlung ohne Wechselwirkung mit den Aerosolteilchen das Volumen passieren kann, ist die Verwendung eines separaten Referenzstrahles nicht nötig (Abb. 3). Es wird nur ein einziger Laserstrahl benötigt, in dessen Verlauf die gebeugten und ungebeugten Wellen interferieren und ein Interferenzmuster bilden, welches aufgenommen und gespeichert werden kann. Dieses Verfahren wird als InlineHolographie bezeichnet.

Vergleich von konventioneller und digitaler Holographie Die konventionelle und die digitale Holographie unterscheiden sich im Wesentlichen durch die Art der Aufnahme und Speicherung des entstehenden Hologramms sowie in der Art der Rekonstruktion des Messobjektes und der weiteren analytischen Bearbeitung. Bei der konventionellen Holographie wird das entstehende Hologramm mit Hilfe von Hologrammplatten aufgenommen, die fotografische Schichten enthalten, die wesentlich

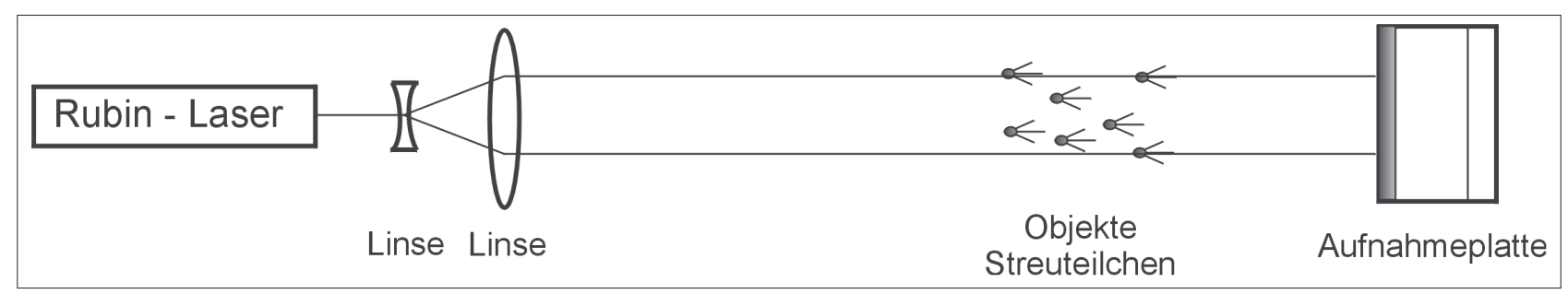

Abb. 3: Prinzipskizze der Anordnung zur Erstellung eines In-Line-Hologramms 
feinkörniger sind als die in der Fotografie verwendeten (Auflösung: 3000 - 5000 Linien/mm). Zur weiteren Verwendung der Hologrammplatte fuir die Rekonstruktion des Objektes muss die Platte nasschemisch entwickelt werden, bevor sie mit dem Referenzstrahl beleuchtet werden kann [11]. Die hohe Feinkörnigkeit der fotografischen Schicht ist im Sinne einer hohen räumlichen Auflösung des Objektes nötig.

Die digitale Holographie verwendet anstelle der fotografischen Hologrammplatte zur Aufzeichnung des entstehenden Hologramms einen elektronischen Sensor in Form einer CCD-Kamera und ein nachgeschaltetes digitales Bildverarbeitungssystem zur Speicherung der Daten. Das Objekt wird nicht in der Sensorebene abgebildet, sondern linsenlos als Fresnel-Hologramm aufgezeichnet. Die Rekonstruktion dieser digital gespeicherten Hologramme kann dann mittels eines Computers numerisch durchgefuihrt werden.

Wesentliche Vorteile der digitalen Holographie gegenüber der konventionellen sind:

- Der Zwischenschritt der Filmentwicklung für die Rekonstruktion des Hologramms entfällt.

- Durch die hohe Abtastrate des Bildaufnahmesystems können dynamische und kinetische Vorgänge untersucht werden.

- Digitale Daten können mit konventionellen Bildverarbeitungsalgorithmen weiterverarbeitet werden.

- Das beobachtbare Volumen ist um eine Größenordnung größer als bei konventionellen Aufnahmetechniken mit Film.

- Digitale Daten können gut dokumentiert und ausgewertet werden.

Die Vorteile der digitalen Holographie machen die Methode der digitalen In-Line-Holographie besonders geeignet für die Untersuchung transparenter Medien, wie z.B. Aerosolen in Luft im Zusammenhang mit der Wolkenbildung und Tröpfchenkinetik in Wolken $[12,13]$.

\subsection{Hologrammerstellung}

\subsubsection{Erstellung von Fresnel-Hologrammen}

Für die Auswahl des Speichermediums ist die Frage des Auflösungsvermögens von sehr großer Bedeutung. Als Auflösungsvermögen bezeichnet man die Fähigkeit des Bildempfängers, die Bildpunkte dicht benachbarter Objekte getrennt aufzuzeichnen. Das Auflösungsvermögen, welches erforderlich ist, um Hologramme aufzuzeichnen, wird bestimmt durch die im Interferenzmuster auftretende maximale Ortsfrequenz. Die Ortsfrequenz ist dabei definiert als räumliche Periodizität in einem Muster. Für die Ableitung der maximalen Ortsfrequenz $\mathrm{f}_{\max }$ in einem Hologramm wird die Interferenz zweier ebener kohärenter Wellen betrachtet (Abb. 4). Diese entsprechen der Referenz- und Objektwelle im holographischen Aufbau. Die Wellen fallen unter den Winkeln $\gamma_{1}$ und $\gamma_{2}$ relativ zum Einfallslot auf die lichtempfindliche Schicht. Der Winkel $\theta_{\max }=\gamma_{1}+\gamma_{2}$ soll der maximal auftretende Winkel zwischen der Referenzwelle und der von einem beliebigen Objektpunkt ausgehenden Objektwelle sein. Die interferierenden Wellen erzeugen ein pe-

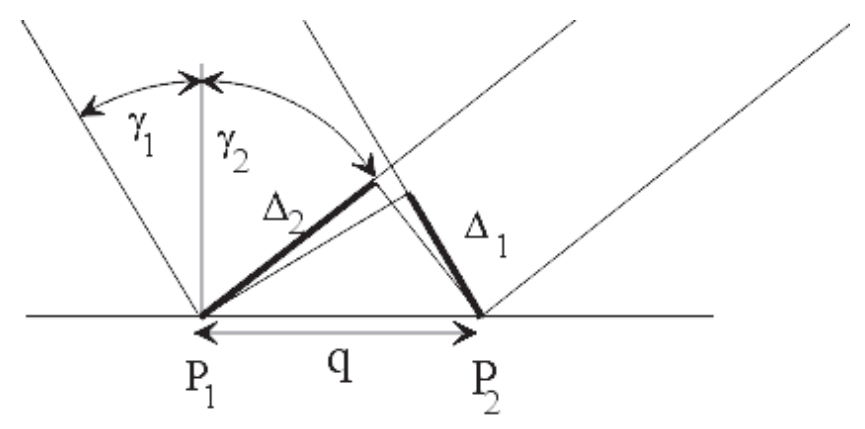

Abb. 4: Schema zur Berechnung der maximalen Ortsfrequenz

riodisches Interferogramm mit der Periodizität q. Die Periodizität q ist der Abstand zweier benachbarter Interferenzmaxima. In der Abb. 4 sind diese Interferenzmaxima mit $P_{1}$ und $P_{2}$ bezeichnet. Die auch als Gangunterschied bezeichnete Differenz der optischen Weglängen beider Bündel beträgt unter diesen Bedingungen eine Wellenlänge.

Es gilt: $\Delta_{1}+\Delta_{2}=\lambda$

Mit $\Delta_{1}=q \sin \gamma_{1}$ und $\Delta_{2}=q \sin \gamma_{2}$

folgt für die räumliche Periodizität:

$q=\frac{\lambda}{\sin \gamma_{1}+\sin \gamma_{2}}$

Für die maximale Ortsfrequenz $\mathrm{f}_{\max }=\mathrm{q}^{-1}$ gilt also unter der Annahme

$\cos \left(\frac{\gamma_{1}-\gamma_{2}}{2}\right) \approx 1$

$f_{\max }=\frac{2}{\gamma} \sin \left(\frac{\gamma_{1}+\gamma_{2}}{2}\right)=\frac{2}{\lambda} \sin \left(\frac{\theta_{\max }}{2}\right)$

Die höchste auftretende Ortsfrequenz wird also durch den maximalen Winkel zwischen der Referenzwelle und der von einem beliebigen Objektpunkt ausgehenden Welle bestimmt. Daher muss man sich bei geringer Auflösung des Speichermediums auf einen kleinen Winkel zwischen Objekt- und Referenzwelle beschränken. Für eine Wellenlänge von $500 \mathrm{~nm}$ und einen größtmöglichen Winkel von $\theta_{\max }=180^{\circ}$ muss eine Ortsfrequenz von 2/ $\mathrm{l}=1 / 250 \mathrm{~nm}^{-1}$ aufgelöst werden. Dieses wird von hochauflösenden Fotoplatten mit einer Auflösung von bis zu 5000 Linienpaaren/mm gewährleistet. Für die digitale Aufzeichnung muss beachtet werden, dass die heutzutage typischen CCD-Kameras Linienpaarabstände von beispielsweise $7 \mu \mathrm{m}$ haben. Die höchsten nach dem Abtasttheorem auflösbaren Ortsfrequenzen $\mathrm{f}_{\max }$ betragen somit $\mathrm{f}_{\max }=(2 \times 7 \mu \mathrm{m})^{-1}$.

Für den maximal zulässigen Winkel zwischen Objektund Referenzwelle gilt dann:

$\theta_{\max }=2 \arcsin \left(f_{\max } \frac{\lambda}{2}\right)$

Mit $\mathrm{l}=514 \mathrm{~nm}$ ergibt sich damit ein Winkel von $\theta_{\max } \approx$ 37 mrad. 


\subsubsection{Erstellung von In-Line-Hologrammen}

Die Verhältnisse sind prinzipiell anders bei der In-LineHolographie, da hier die maximale Ortsfrequenz durch Beugung der einfallenden Wellenfront an einzelnen undurchsichtigen Streuteilchen im sonst lichtdurchlässigen Objekt bestimmt wird. Die Beugung an einem kleinen Streuteilchen vom Durchmesser $\rho$ ist nach dem Theorem von Babinet äquivalent der Beugung an einer Lochblende mit dem Lochdurchmesser r. Daher gilt für den Beugungswinkel $\theta_{\max }$ in die \pm 1 . Beugungsordnung:

$\sin \theta_{\max }=1,22 \frac{\lambda}{\rho}$

wobei gilt: $\rho=r$

Auf dem im Abstand $\mathrm{z}$ angeordneten Hologramm muss mindestens die erste Beugungsordnung aufgenommen werden, um das Objekt aus dem Hologramm rekonstruieren zu können. Es gilt also:

$$
\sin \theta_{\text {max }} \approx \tan \theta_{\text {max }}=\frac{q}{z}
$$

Der Abstand q der ersten Beugungsordnung von der optischen Achse kann als ein Maß für die räumliche Periodizität des Beugungsbildes aufgefasst werden. Daher folgt

$$
q=\frac{\lambda z}{\rho}
$$

und für die maximale Ortsfrequenz

$$
f_{\max }=q^{-1}=\frac{\rho}{\lambda z}
$$

Um ein In-Line-Hologramm aufzuzeichnen, müssen vom Aufzeichnungsmedium also lediglich die Beugungsstrukturen von einzelnen Teilchen im Objektvolumen aufgelöst werden. Anders als bei der Fresnel-Holographie geht der Winkel zwischen Objekt- und Referenzwelle hier nicht in die Berechnungen ein.

Der Prozess der Erstellung und Aufzeichnung eines InLine-Hologramms für den Fall einer Aerosolpartikel-Verteilung ist in der Abb. 5 dargestellt. Die Strahlung eines
Rubinlasers ist durch ein Teleskop aufgeweitet und kollimiert zu einem parallelen Strahl mit großem Durchmesser, der das Messvolumen durchstrahlt. Ein Teil der Laserstrahlung wird an den transparenten, halbtransparenten und undurchsichtigen Partikeln gestreut und gebeugt und bildet die Objektwelle, der andere Teil der Strahlung passiert das Untersuchungsvolumen ungehindert. Er bildet die Referenzstrahlung. Die Überlagerung beider Teilwellen in der Aufzeichnungsebene erzeugt das In-Line-Hologramm, das alle Informationen bezüglich Partikelgröße, Partikelverteilung, Partikelposition u. a. in durch Phasenbeziehungen und Lichtintensität codierter Form enthält. Das Hologramm ist entweder im Rahmen der herkömmlichen Holographie auf einem geeigneten Film oder bei der digitalen Holographie durch einen optoelektronischen Sensor mit nachgeschaltetem Rechner gespeichert.

Um den gegenüber mechanischen Bewegungen empfindlichen Messaufbau weitgehend unempfindlich zu machen, wird ein Rubinlaser mit sehr kurzer Impulsdauer (Pulslänge 30 ns) verwendet.

\subsection{Rekonstruktion des Objektes}

\subsubsection{Rekonstruktion bei konventioneller Holographie}

Zur Rekonstruktion des Objektes wird das gewonnene Hologramm nur noch mit der Referenzwelle beleuchtet. Dabei wird diese an der Struktur des Hologramms gebeugt. Eine der Beugungsordnungen ist die urspruingliche Objektwelle, die vom Objekt herzukommen scheint (Abb. 2) [10]. Das auf diese Weise entstandene virtuelle Bild kann dann entweder durch die Augen abgebildet oder z. B. durch eine elektronische Kamera aufgenommen werden. Auf diese Weise steht ein dreidimensionales Bild des Objektes zur Verfuigung.

Mit vollautomatisierter Bildanalyse kann das rekonstruierte Messvolumen Ebene für Ebene mit einem kurzbrennweitigen Optiksystem dreidimensional abgetastet werden. Die Daten werden an eine Auswertesoftware weitergeleitet.

Ein anderes, häufig verwendetes Rekonstruktionsverfahren ist in der Abb. 6 dargestellt. Dieses Verfahren kommt besonders bei der Messung von Aerosolen in

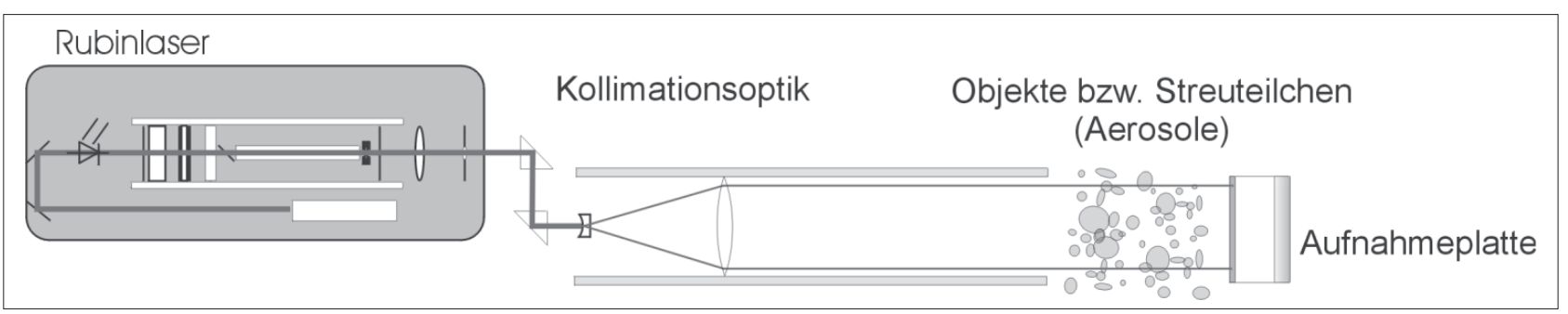

Abb. 5: Prinzipskizze der Anordnung zur Erzeugung und Aufzeichnung eines In-Line-Hologramms von Aerosolverteilungen

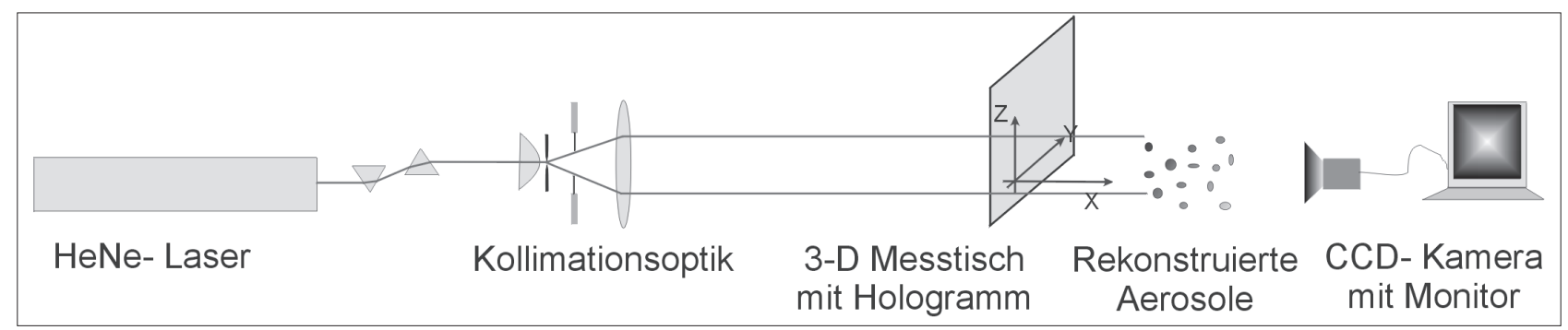

Abb. 6: Prinzipskizze der Anordnung zur Rekonstruktion des Hologramms einer Aerosolverteilung mit CCD-Kamera und Präzisionsverstellung der Hologrammplatte 
durchsichtigen Messvolumina zur Anwendung. Bei diesem Verfahren ist das Hologramm auf einem in 3 Achsen verschiebbaren Präzisionsmesstisch senkrecht zur Strahlungsrichtung eines HeNe-Lasers fixiert. Durch Beleuchtung des Hologramms mit Laserstrahlung wird das gespeicherte Messvolumen hinter der holographischen Platte rekonstruiert. Eine CCD-Kamera mit stark vergrößerndem Objektiv macht die Aerosolpartikel im rekonstruierten Messvolumen sichtbar. Durch systematische Verschiebung der Hologrammplatte im Laserstrahl ist es möglich, definierte Volumina im Messvolumen näher zu untersuchen (Abb. 7)

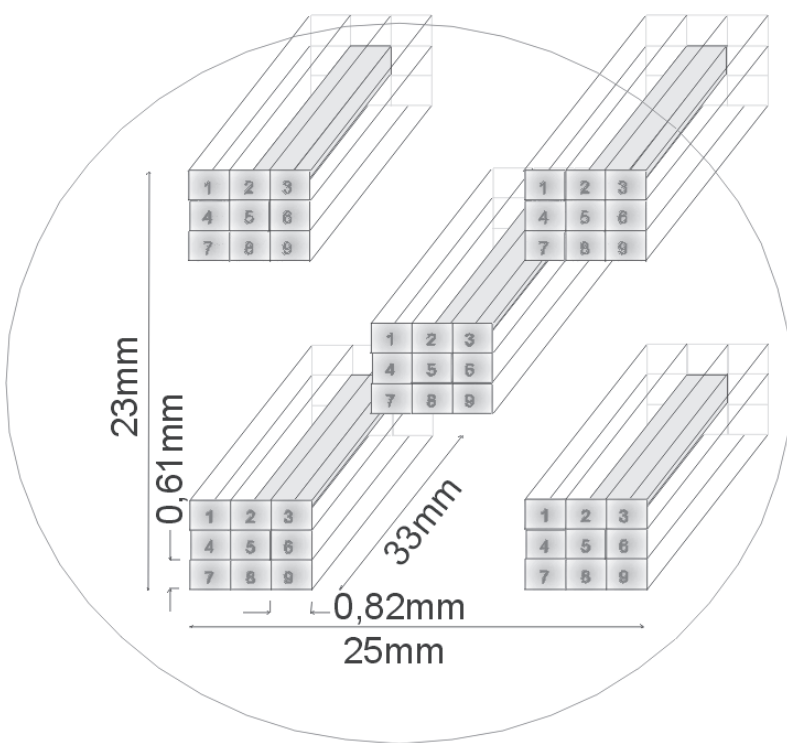

Abb. 7: Schema für die Auswertung eines rekonstruierten Messvolumens

\subsubsection{Rekonstruktion bei digitaler Holographie}

Bei der digitalen Holographie wird die Objektrekonstruktion numerisch mit Hilfe eines Computers durchgeführt. Dabei wird die diskrete Form der Fraunhofer-Beugung auf das digital gespeicherte Hologramm angewendet [14]. Das Ergebnis dieser algorithmischen Operation ist eine spezielle, numerisch rekonstruierte Ebene aus dem gesamten betrachteten Messvolumen. Die Amplitudenverteilung in der rekonstruierten Ebene wird durch den folgenden Ausdruck (Gleichung (10)) beschrieben:

$\Gamma(m, n)=\frac{i a}{\lambda z} \exp \left[-i \pi \lambda z\left(\frac{m^{2}}{N^{2} \Delta x^{2}}+\frac{n^{2}}{N^{2} \Delta y^{2}}\right)\right]$

$* \sum_{k=0}^{N-1} \sum_{l=0}^{N-1} T(k, l) \exp \left[-i \frac{\pi}{\lambda z}\left(k^{2} \Delta x^{2}+l^{2} \Delta y^{2}\right]\right.$

$\exp \left[+i 2 \pi\left(\frac{k m}{N}+\frac{\ln }{N}\right)\right]$

$\Gamma(\mathrm{m}, \mathrm{n})$ diskrete, rekonstruierte Ebene mit den Bildelementen $\mathrm{x}$ und $\mathrm{y}$

$\mathrm{T}(\mathrm{k}, \mathrm{l})$ auf dem CCD-Chip gespeichertes Hologramm mit den Koordinaten $\mathrm{x}$ und y (reale Interferenzstruktur)

$\lambda$ benutzte Wellenlänge

z Koordinatenpunkt für die Rekonstruktionsebene $\Gamma(\mathrm{m}, \mathrm{n})$ entlang der Beobachtungsrichtung

$\Delta \mathrm{x}, \Delta \mathrm{y} \quad$ Pixelgröße des CCD-Chips
Gleichung (10) gilt allgemein für ebene Referenzwellen, die senkrecht auf die Hologrammebene fallen. Sie gilt also auch fuir den Spezialfall der In-Line-Holographie.

Eine dreidimensionale Bearbeitung des Messvolumens wird durch eine Berechnung von rekonstruierten Ebenen bei verschiedenen Positionen entlang der Beobachtungsrichtung $\mathrm{z}$ erreicht. Durch Wahl genuigend kleiner $\mathrm{Ab}$ stände $\Delta \mathrm{z}=\mathrm{z}_{1}-\mathrm{z}_{2}$ zwischen den aufeinanderfolgenden Ebenen ist eine ebenenweise Zusammensetzung des rekonstruierten Messvolumens möglich.

\subsection{Hologrammauswertung}

Für eine räumliche Auflösung des Messvolumens z. B. im Sinne einer partiellen Untersuchung des Volumens auf einzelne Aerosolpartikel wird das Hologramm, wie im Kap. 2.3.1 beschrieben, auf einem 3-D-Messtisch fixiert und verschoben. Das gespeicherte Messvolumen wird dann bei Beleuchtung des Hologramms mit Laserlicht hinter der Hologrammplatte rekonstruiert. Eine CCDKamera mit stark vergrößerndem Objektiv (Gesamtvergrößerung $M=270$ ) macht die Aerosolpartikel sichtbar. Durch Verschiebung des Hologramms wird dann ein definiertes Volumen auf Aerosole systematisch untersucht. In der Abb. 7 ist die Prozedur einer Volumenanalyse schematisch dargestellt.

\section{Messsystem}

Laborexperimente haben gezeigt, dass mit Hilfe der konventionellen Holographie Aerosolpartikel im Größenbereich 5 - 500 mm rekonstruierbar sind. Dieser Größenbereich ist unter anderem für die Wolkenphysik, speziell für die Untersuchung der Kinetik von Wolkentröpfchen interessant. Für diesen Zweck wurde ein In-Line-Holographiesystem entwickelt, dessen Funktionsprinzip im Kapitel 3.2.2 erläutert und sein Aufbau in der Abb. 5 dargestellt ist. Dieses System wurde nach einer Laborerprobungsphase in der Zeit vom 28.09.1998 bis 09.10.1998 für Messzwecke in der Atmosphäre auf dem Brocken/Harz in einer Messkampagne unter extremen Bedingungen eingesetzt.

\section{Messergebnisse}

Der Brocken war während der Messkampagne fast ausschließlich in Wolken gehüllt. Die Temperaturen lagen im Bereich zwischen $-6{ }^{\circ} \mathrm{C}$ und $+5{ }^{\circ} \mathrm{C}$. Die Windgeschwindigkeit betrug etwa $12 \mathrm{~m} / \mathrm{s}$ und war in Böen beträchtlich höher. Unter diesen extremen Wetterbedingungen wurden Wolkenvolumina in Hologrammen aufgezeichnet und gespeichert. Nach der Filmentwicklung konnten Wolkenvolumina in der trockenen und geschuitzten Umgebung eines Labors auf der Brockenstation rekonstruiert und auf ihre Partikelverteilung untersucht werden. In der Abb. 7 ist für ein Hologramm vom 07.10.1998 die Geometrie des ausgewerteten Volumens und das Schema der Analysenprozedur dargestellt. Die Größe des untersuchten Wolkenvolumens 


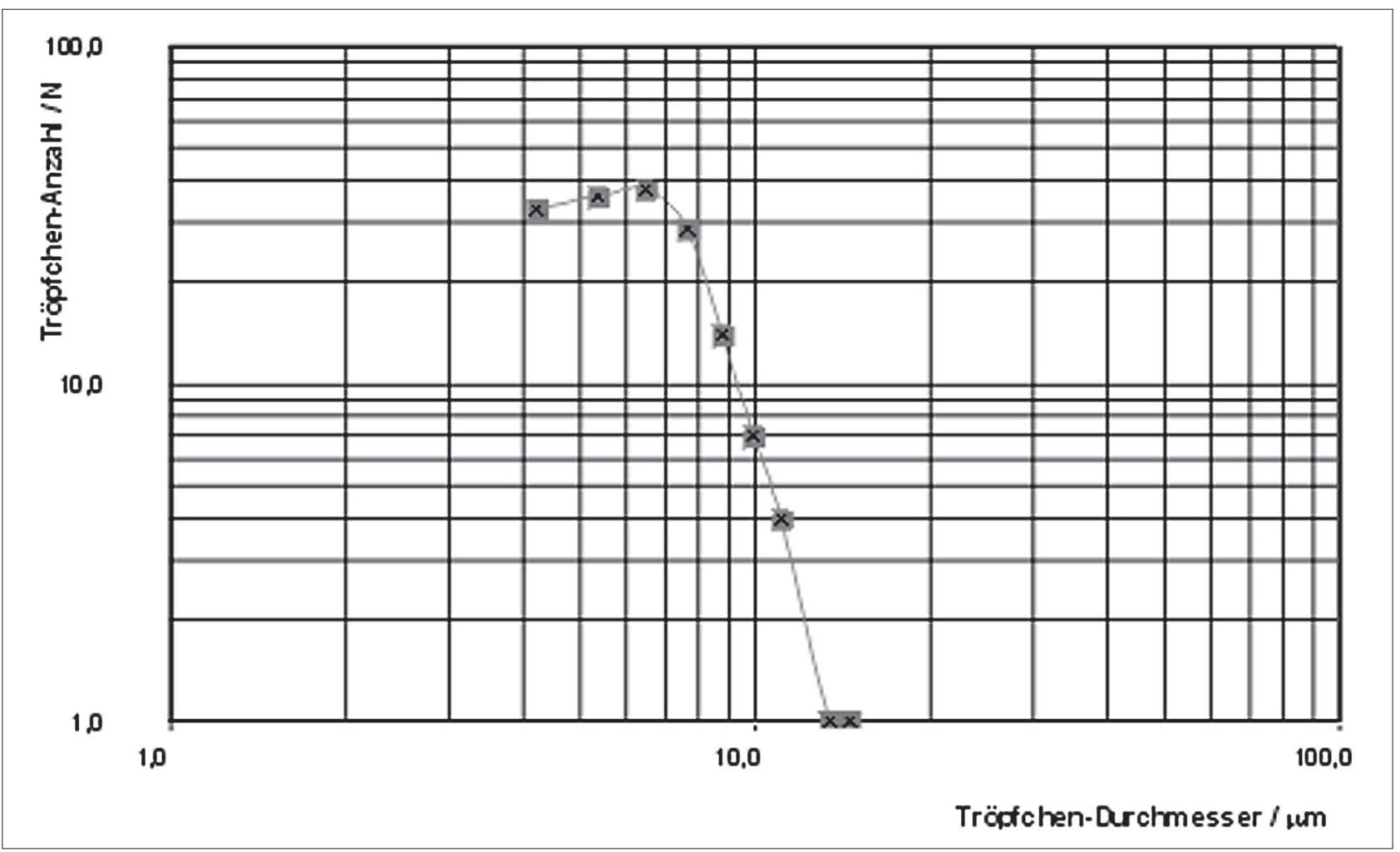

Abb. 8: Tröpfchengrößenverteilung in einem Wolkenvolumen vom 07.10.1998

betrug $0,864 \mathrm{~cm}^{3}$, die Tröpfchendichte $\rho_{\mathrm{T}}=188 \mathrm{~cm}^{-3}$. Die zugehörige Tröpfchengrößenverteilung zeigt die Abb. 8 , die entsprechende Balkendiagrammdarstellung die Abb. 9. Der mittlere Teilchenabstand betrug $\mathrm{d}_{\mathrm{m}}=$ $1,74 \mathrm{~mm}$.

Einen Tag später, am 08.10.1998, war in den Wolken zwar der Tröpfchengrößenbereich von 4 - 16 mm gegenüber dem Vortag weit gehend identisch, die Verteilung der Tröpfchengröße hatte sich jedoch, wie aus der Abb. $10 \mathrm{zu}$ ersehen ist, verändert. Bei einer größeren Tröpfchendichte von $\rho_{\mathrm{T}}=266 \mathrm{~cm}^{-3}$ und kleinerem mittleren Teilchenabstand $\mathrm{d}_{\mathrm{m}}=1,55 \mathrm{~mm}$ hatte sich das Teilchengrößenspektrum von kleineren zu größeren Teilchen verschoben. Dieses Phänomen ist sicher u. a. eine Folge der höheren Tröpfchendichte und damit der höheren Koagulationswahrscheinlichkeit.

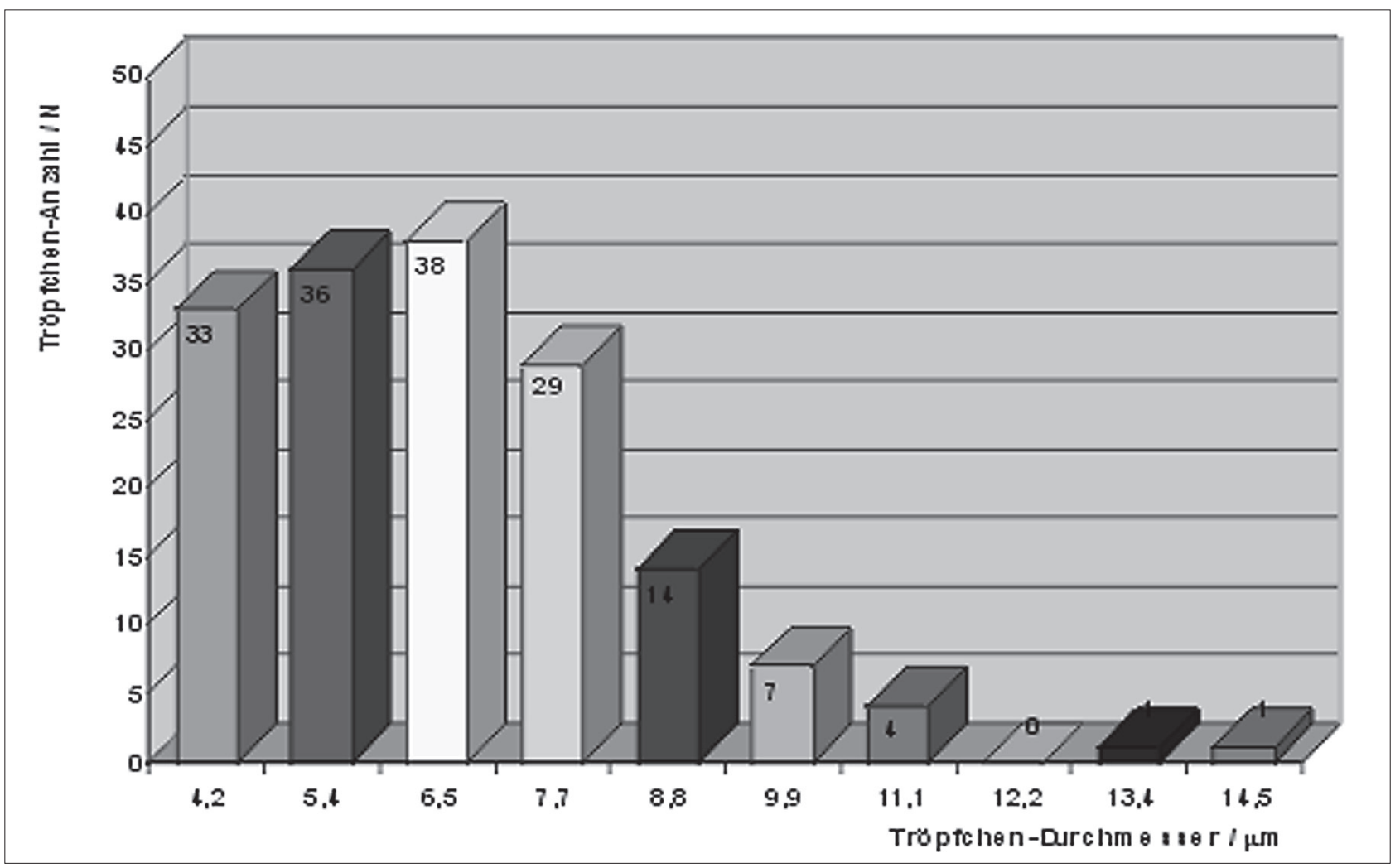




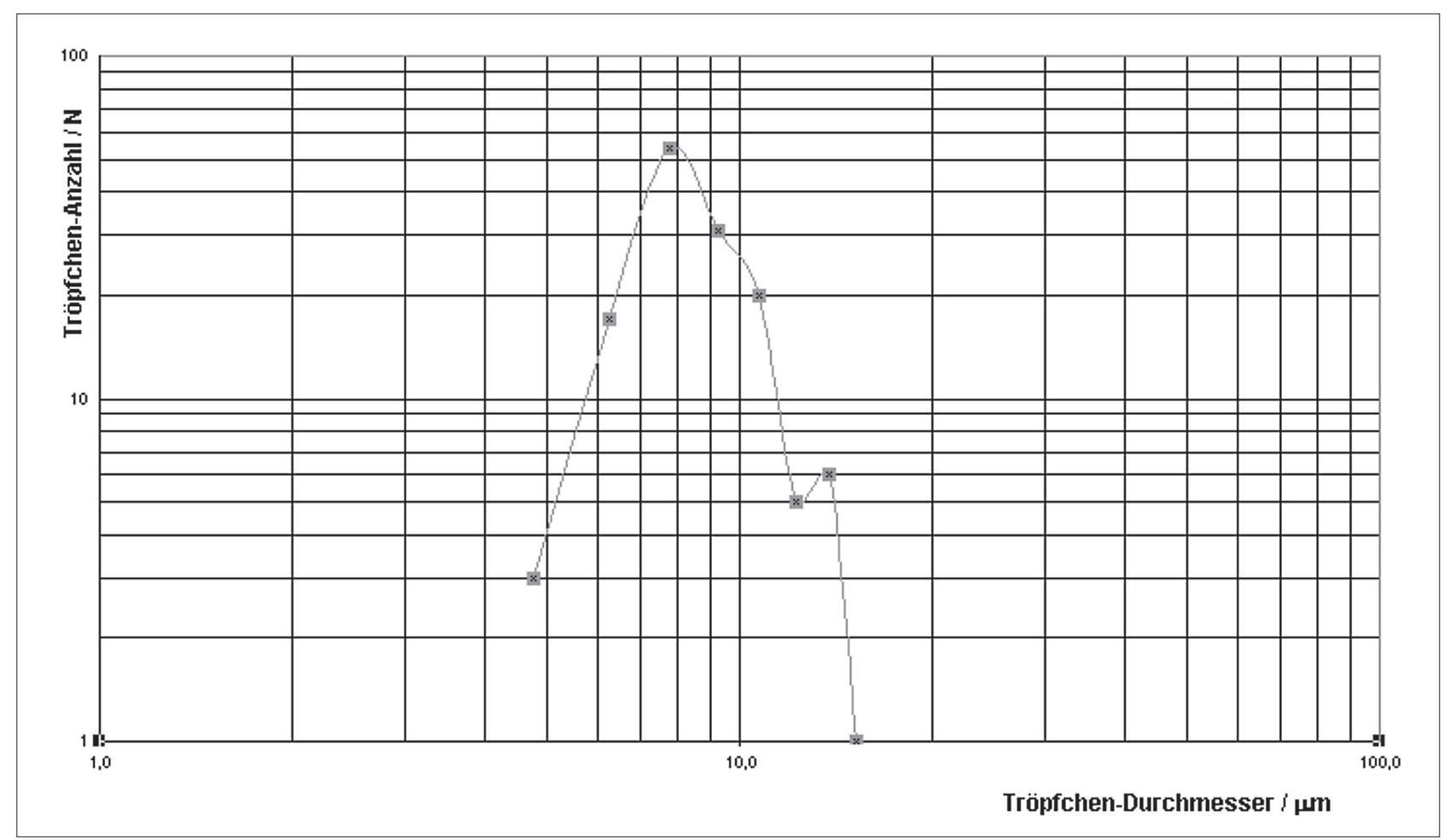

Abb.10: Tröpfchengrößenverteilung in einem Wolkenvolumen vom 08.10.1998

\section{Schlussfolgerungen}

Mit konventionellen holographischen Techniken unter Verwendung von holographischen Platten oder Filmen sind Teilchengrößen bis zu einem Durchmesser von $3 \mu \mathrm{m}$ rekonstruierbar. Bei Anwendung kurzwelligerer Laserstrahlung $\left(\lambda<\lambda_{\mathrm{HeNe}} 632,8 \mathrm{~nm}\right)$ für die Hologrammerzeugung und Rekonstruktion sind Partikel bis zu einem Durchmesser von $1 \mu \mathrm{m}$ auflösbar.

Bei Experimenten mit der digitalen holographischen Methode wurden mit einer CCD-Kamera mit $756 \times 581$ Pixeln Partikelgrößen von 20 mm identifiziert. Mit Hilfe von CCD- Kameras mit $4048 \times 4048$ Pixeln und kleineren Pixelgrößen von nur $9 \times 9 \mu \mathrm{m}^{2}$ sind bezüglich der Partikelgrößen-Auflösung verbesserte Rekonstruktionsergebnisse bis zu einem Faktor 64 zu erwarten. Die erreichbare Auflösung kann durch optische Vorvergrößerung des Messvolumens vor der Aufzeichnung noch verbessert werden.

\section{Literatur}

[1] Hobbs, P. V.: Aerosol-Cloud-Climate Interactions. Academic Press, Inc. 1993.

[2] Jäger, H.: Lidar observed trend in stratospheric background aerosol. Technical Digest on Optical Remote Sensing of the Atmophere. Vol. 18, Washington D. C., pp. 153 - 155, (1991).

[3] Charlson, R. J. u. a.: Climate forcing by anthropogenic aerosols. Science 255, pp. 423 . 430, (1992).

[4] Langner, J. u. a.: Anthropogenic influence on the distribution of tropospheric sulphate aerosol. Nature 359, pp. 712 - 716, (1992).
[5] Graßl, H.: The influence of aerosol particles on radiative parameters of clouds. Idöjaras, Band 86, S. 60 - 74, (1992).

[6] Kiehl, J. T.; Bringleb, B. P.: The relative roles of sulfate aerosol and greenhouse gases in climate forcing. Science 260, pp. 311 - 314, (1993).

[7] Jaenicke, R.; Hanusch, T.: Simulation of the Optical Particle Counter Forward Scattering Spectrometer Probe 100 (FSSP-100). Aerosol Science and Technology 18, pp. 309 - 322, (1993).

[8] Swithherow, W. K.: A high resolution holographic particle sizing system, Opt. Eng. 18, No. 3, (1979).

[9] Prikryl, I., et. al.: Holographic imaging of semitransparent droplets or particles. Appl. Optics. 21, (1982).

[10] Fouckhardt, H.: Photonik. Eine Einfuihrung in die integrierte Optoelektronik und technische Optik. B. G. Teubner, Stuttgart 1998.

[11] Vössig, H. u. a.: HODAR holography applied to raindrop and snowflake in-situ measurements. J. Aerosol Sci. $\underline{28}$, Suppl. 1, pp. 375 - 376, (1997).

[12] Vikram, Ch. S.: Particle Field Holography. Cambridge University Press 1992.

[13] Borrmann, S.; Jaenicke, R.: Instrument Intercomparison Study on Cloud Droplet Size Distribution Measurements. J. Atmosph. Chem. 19, pp. 253 - 258, (1994).

[14] Jüptner, W.; Osten, W.: Fringe '97, Proceedings of the 3th Int. Workshop on Automatic Processing of fringe patterns. Academy press, optical metrology $\underline{3}$,), pp. 353 363, (1997). 


\section{Autoren}

\section{Felix Frischkorn}

ELAS Emder Lasertechnik

Zum Nordkai 16

26725 Emden

Tel. (0 49 21) 996807

Fax (0 49 21) 996808

E-Mail: frischkorn@emder-lasertechnik.de

\section{Prof. Dr. Horst Kreitlow}

Jörn Miesner

Fachhochschule Ostfriesland Emden

Fachbereich naturwissenschaftliche Technik

Institut für Lasertechnik Ostfriesland (ILO)

Constantiaplatz 4

26723 Emden

Tel. (0 49 21) 8071603

Fax (0 49 21) 8071593

E-Mail: kreitlow@nwt.fho-emden.de

\section{Dr. habil. Lothar Martini}

Prof. Dr. sc. nat. Bernd Stark

Technische Fachhochschule Wildau

Fachbereich Ingenieur-/Wirtschaftsingenieurwesen

Labor für Sensortechnik/Gasanalytik

Tel. (0 33 75) 504014

Fax (0 33 75) 553876

E-Mail: bstark@igw.tfh-wildau.de

\section{Dr.-Ing. Roland Wernecke}

Deka Sensor + Technologie

Entwicklungs- und Vertriebsgesellschaft $\mathrm{mbH}$

Potsdamer Straße 18a

14513 Teltow

Tel. (0 33 28) 430250 